\title{
Multi-Objective Multi-Dimensional Transportation: A Case Study to the Flow of the Commodities of the Main Roads to Main Nodes in the North Western Coastal Strip of Egypt
}

\author{
Taghreed Abo-Kila1, Yousria Abo-Elnaga², Abd Allah A. Mousa ${ }^{3,4}$ \\ ${ }^{1}$ Department of Geography, Faculty of Arts, Benha University, Banha, Egypt \\ ${ }^{2}$ Department of Mathematics, Higher Technological Institute, Tenth of Ramadan City, Egypt \\ ${ }^{3}$ Department of Basic Engineering Science, Faculty of Engineering, Menoufia University, Shebin El-Kom, Egypt \\ ${ }^{4}$ Department of Mathematics and Statistics, Faculty of Sciences, Taif University, Taif, KSA \\ Email: a_mousa15@yahoo.com
}

How to cite this paper: Abo-Kila, T., Abo-Elnaga, Y. and Mousa, A. (2021) Multi-Objective Multi-Dimensional Transportation: A Case Study to the Flow of the Commodities of the Main Roads to Main Nodes in the North Western Coastal Strip of Egypt. Journal of Geographic Information System, 13, 353-368.

https://doi.org/10.4236/igis.2021.133020

Received: August 1, 2019

Accepted: June 27, 2021

Published: June 30, 2021

Copyright $\odot 2021$ by author(s) and Scientific Research Publishing Inc. This work is licensed under the Creative Commons Attribution-NonCommercial International License (CC BY-NC 4.0). http://creativecommons.org/licenses/by-nc/4.0/ (c) (i) (s) Open Access

\begin{abstract}
The distribution of merchandises and commodities from source towns to final destinations is a vital issue. The job of transporter's decisions can be optimized by reformulating the transportation problem as generalization of the classical transportation problems. Multiobjective multi-dimensional transportation network is considered the extension of conventional two-dimensional transportation network and is convenient for dealing with transportation systems with multiple supply nodes, multiple demand nodes, as well as diverse modes of transportation demands or delivering multiple kinds of merchandises. In this study, we implement an improved Biogeography based optimization IBBO to the flow of the commodities of the main roads to main nodes in the North Western Coastal Strip of Egypt, where there are four main roads and three nodes. The proposed algorithm incorporates the dominance criteria to handle multiple objective functions which enable the decision maker to cover all the Pareto frontier of the problem which have a large-scale size. Numerical results were reported in order to establish the real computational burden of the proposed algorithm and to assess its convergence performances for solving real geographical problem.
\end{abstract}

\section{Keywords}

Biogeography Based Optimization, Multiobjective Optimization, Multi-Dimensional Transportation 


\section{Introduction}

The transportation network system is a special kind of general Linear Programming Problems LPs. Recently, the transportation network has been widely investigated in logistics, mathematics, transportation economics, and operations systems where distribution and transportation of commodities from town sources to final destinations is a vital issue [1]. The job of transporter's decisions can be optimized by reformulating the transportation Problem as generalization of the classical transportation network [2] [3].

The conventional transportation network can be formulated as a mathematical model which optimizes the cost objective function satisfying certain constraints. In two dimensional problems, transporting goods from $\mathrm{m}$ sources to $\mathrm{n}$ destinations is to be minimized. Multi-dimensional transportation network [4] [5] is the large-scale extension of small classical transportation networks and is convenient for dealing with transportation problems with different supply nodes, different demand nodes as well as problems having multiple modes of transportation or delivering different kinds of goods. Thus, the proposed problem would be a more complex and large-scale problem than conventional transportation problems.

Nature based Inspired Algorithms (NIAs) [6] [7] are the main source for improving intelligent systems. It also enables the decision maker to get the solutions for difficult, large-scale, and non-formulated mathematical problems. A function is optimized by Nature-based-Inspired-Algorithms by creating a candidate agent in terms of the measure of fitness (objective function). The distinguished characteristics of biogeography are maintained by Biogeography based optimization (BBO) [8] [9]. Biological species can be classified according to space, and time by researching biogeography. In BBO, solution features are migrated between species which are motivated by the scientific model of biogeography.

This paper presents an improved Biogeography based optimization IBBO for solving multiobjective multi-dimensional transportation problem. The proposed approach was implemented to the flow of the commodities of the main roads to main nodes in the North Western Coastal Strip of Egypt, where there are four main roads and three nodes. Numerical results were reported in order to set up real numerical burden of the proposed system and to assess its convergence performances for solving real geographical transportation problem.

This paper is organized as follows: Optimal Transportation-Based Graph network is presented in Section 2. The Classical Transportation Problems are described in Section 3. Multiple Objective Transportation Problem is presented in Section 4. Multi-dimensional Bi-Criterion Transportation Problem is discussed in Section 5. Relaxed Multi-dimensional multi-objective transportation problem is introduced in Section 6. Section 7 presents a Modified BBO algorithm. Experimental numerical results and discussions are discussed in Section 8. Finally, the conclusion is investigated in Section 9. 


\section{Optimal Transportation-Based Graph Network}

The analysis and solution of the multidimensional transportation problem [10] indicate whether a certain road should be used for transporting commodities from the perspective of optimizing transportation costs and, equivalently, if a connection between landmarks $\mathrm{n}_{1}$ and $\mathrm{n}_{2}$ should be included in the shape representation of the network. Figure 1 shows the main roads and main nodes in the north western coastline [3].

\section{Classical Transportation Problem}

The aim of the Classical Transportation network is to minimize the routing cost of goods from the supply node to the destination node. The general transportation problem can be represented as: each good is available at each of $m$ sources and it is required that given quantities of the product be transported to each of $n$ final destinations. The minimum cost of transporting a unit of each product from any source to any destination is known. The transportation schedule which minimizes the total cost of shipment is to be determined [11]. The single objective, two-dimensional transportation problem can be formulated as:

$$
\begin{aligned}
& \text { Minimize } \quad z=\sum_{i=1}^{m} \sum_{j=1}^{n} C_{i j} x_{i j}, \\
& \text { Subject to } \\
& \qquad \sum_{j=1}^{n} x_{i j}=a_{i}, \quad i=1,2, \cdots, m \\
& \sum_{i=1}^{m} x_{i j}=b_{j}, \quad j=1,2, \cdots, n \\
& x_{i j} \geq 0 \quad \forall i, j
\end{aligned}
$$

$x_{i j}$ : The quantity of the product to be transported from origin $i$ to destination $j$.

$a_{i}$ : The available quantity at source $i$.

$b_{j}$ : The required quantity at destination $j$.

$c_{i j}$ : The unit cost of transporting from source $i$ to destination $j$.

The goal of transportation problem is to minimize the cost function which satisfies the constraints. The traditional objective cost is one of minimizing the total transportation cost.

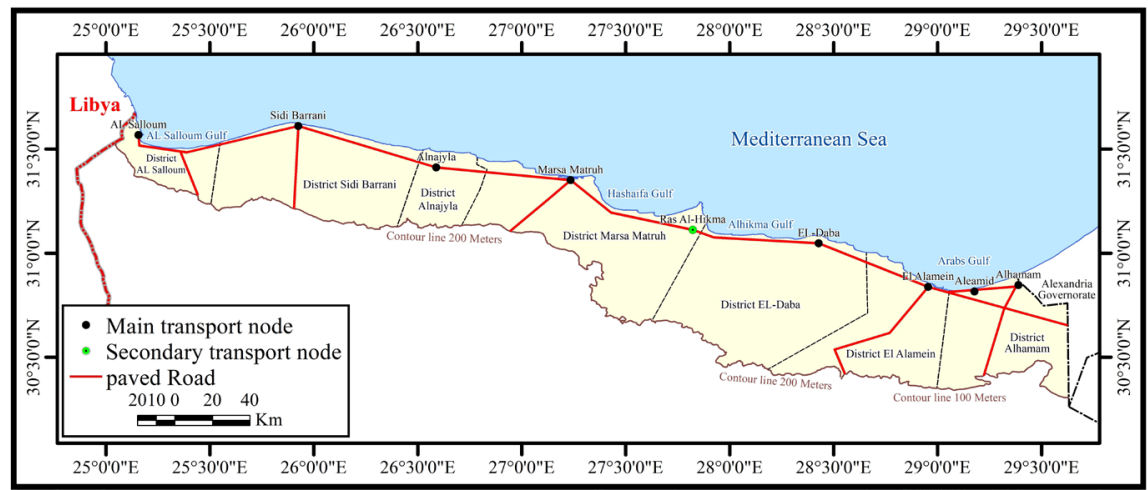

Figure 1. The topological shape of the road networks in the north-western coastline [3]. 


\section{Multiple Objective Transportation Problem}

Multiple objective transportation optimization problem [12] can be defined as: "A set of decision variables which optimizes a vector cost functions which represent the cost functions, also satisfying the constraints". Hence, the terminology "optimize" means to find a solution which would return the minimum cost values of all the cost functions acceptable to the decision maker Kasana and Kumar [13] formulated the multi-objective transportation two-dimensional problem as follows:

$$
F(x)=\left\{\begin{array}{c}
F_{1}=\sum_{i=1}^{m} \sum_{j=1}^{n} C_{i j}^{1} x_{i j}, \\
F_{2}=\sum_{i=1}^{m} \sum_{j=1}^{n} C_{i j}^{2} x_{i j}, \\
\cdots \\
F_{l}=\sum_{i=1}^{m} \sum_{j=1}^{n} C_{i j}^{l} x_{i j}
\end{array}\right\}
$$

Subject to

$$
\begin{aligned}
& \sum_{j=1}^{n} x_{i j}=a_{i}, \quad i=1,2, \cdots, m \\
& \sum_{i=1}^{m} x_{i j}=b_{j}, \quad j=1,2, \cdots, n \\
& x_{i j} \geq 0 \quad \forall i, j
\end{aligned}
$$

$c_{i j}^{l}, i=1,2, \cdots, m ; j=1,2, \cdots, n$, is the required unit cost needed for shipping one unit of the product quantity from source $i$ to the destination $j$ satisfying $l$ objective functions.

Let us consider $m$ sources, $O_{1}, \cdots, O_{m}$ and $n$ destination, $D_{1}, \cdots, D_{n}$. Further, the cost of shipping one unit of goods from $i^{\text {th }}$ plant to $f^{\text {th }}$ destination is $C_{i j}^{l}: i=1, \cdots, m ; j=1, \cdots, n$. The necessary and sufficient condition for the existence of feasible solution to balanced classical transportation problem is as follows:

$$
\sum_{i=1}^{m} a_{i}=\sum_{j=1}^{n} b_{j}
$$

The constraint $\sum_{j=1}^{n} x_{i j}=a_{i}, i=1,2, \cdots, m \& \sum_{i=1}^{m} x_{i j}=b_{j}, j=1,2, \cdots, n$ represent $m+n$ equations in $m \times n$ positive variables. Each variable $x_{i j}$ appears in exactly two constraints, one is associated with source and other with destination.

\section{Representation of Multi-Dimensional Bi-Criterion Transportation Problem}

A conventional transportation network can be formulated in the form of a two-dimensional table for $i=1,2, \cdots, m \& j=1,2, \cdots, n$ as illustrated in Figure 2. In this figure, each cell illustrates one value of the $x_{i j}$ 's. When these values $x_{i j}$ 's are summed along the rows of the table they must equal $b_{i}$, and when they are summed down the columns they must equal $a_{i}$. 


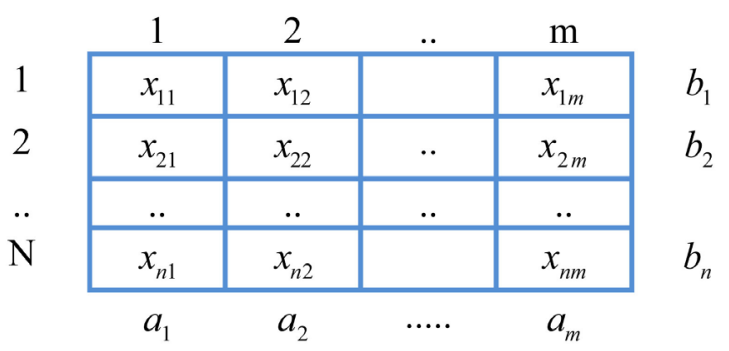

Figure 2. Two dimensional transportation problem.

\subsection{Graphical and Mathematical Representation of Three-Dimensional Transportation Problem}

Three index multi-dimensional transportation problem is a natural extension of the two-dimensional transportation type, which is explained by Haley [14]. The multi-dimensional transportation problem in which there are $m$ different sources, $n$ different destinations and $p$ of commodities type to be shipped can be represented as follows.

$$
\begin{aligned}
\operatorname{minimize}\left\{z_{1}\right. & =\sum_{i=1}^{m} \sum_{j=1}^{n} \sum_{k=1}^{p} C_{i j k} x_{i j k}, \\
z_{2} & \left.=\max \left\{t_{i j k}: x_{i j k}>0,(i=1,2, \cdots, m ; j=1,2, \cdots, n ; k=1,2, \cdots, p)\right\}\right\}
\end{aligned}
$$

Subject to

$$
\begin{aligned}
& \sum_{i=1}^{m} x_{i j k}=A_{j k}, \sum_{j=1}^{n} x_{i j k}=B_{k i}, \sum_{k=1}^{p} x_{i j k}=E_{i j} \\
& x_{i j k} \geq 0 ; i=1,2, \cdots, m ; j=1,2, \cdots, n ; k=1,2, \cdots, p \\
& \sum_{j=1}^{n} A_{j k}=\sum_{i=1}^{m} B_{k i}, \sum_{k=1}^{p} B_{k i}=\sum_{j=1}^{n} E_{i j}, \sum_{j=1}^{m} E_{i j}=\sum_{i=1}^{p} A_{j k} \\
& \sum_{j=1}^{n} \sum_{k=1}^{p} A_{j k}=\sum_{k=1}^{p} \sum_{i=1}^{m} B_{k i}=\sum_{i=1}^{m} \sum_{j=1}^{n} E_{i j}
\end{aligned}
$$

Three index multi-dimensional transportation problems may be represented as a block diagram, in which the layers in all directions form restricted transportation problem which is declared in Figure 3 . The solid problem can be set out as a three-dimensional block for $i=1,2, \cdots, m \& j=1,2, \cdots, n \& k=1,2, \cdots, p$, in which, each cell represents one of the $x_{i j k}$ 's. When these values $x_{i j k}$ 's are summed along the rows (constant $j$ and $k$ ) they equal $A_{j k}$, also, when these values are summed along the columns (constant $k$ and $i$ ) they equal $B_{k i}$, finally, when these values $x_{i j k}$ 's are summed down the heights (constant $i$ and $j$ ) they equal $C_{i j}$.

\subsection{Mathematical Representation of Four-Dimensional Transportation Problem}

The mathematical representation of four-dimensional transportation problem is stated according to previous description. For easy representation, all basic notions will be illustrated and defined as follows: 


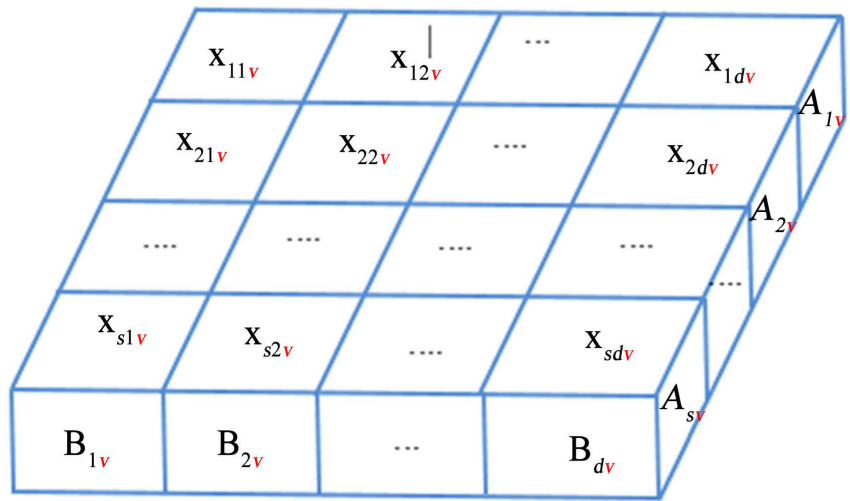

Vehicle mode $\mathbf{v}$

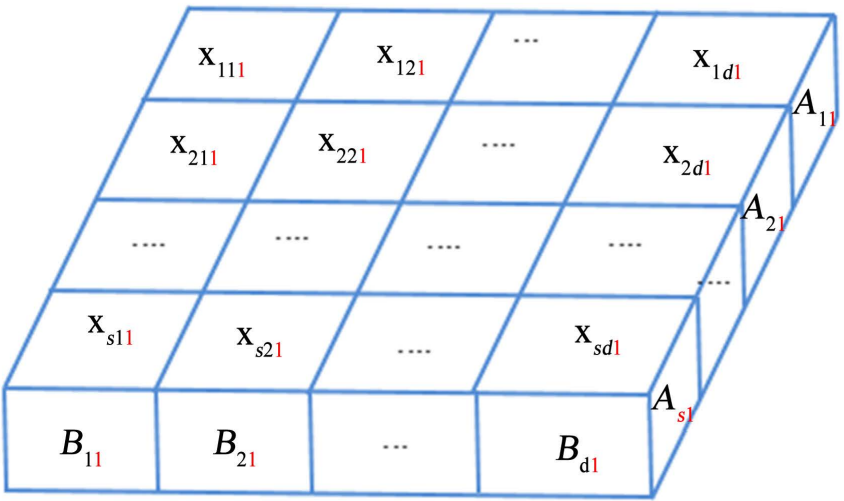

Vehicle mode 1

Figure 3. Three index multidimensional transportation problem with $m$ different sources and $n$ different destinations and $\mathrm{v}$ different vehicles [15].

$i=1,2, \cdots, m$ are $m$ origins which represent the main sources sites.

$j=1,2, \cdots, n$ are $n$ destinations locations where the products are transported to them.

$k=1,2, \cdots, p$ represent the type of vehicle (means of transportation).

$l=1,2, \cdots, p$ represent the types of the transported product.

$A_{i k l}$ is the quantity of shipped goods from the origin $i$ by vehicle $k$ with a product $I$

$B_{j k l}$ is the quantity to be shipped to destination $j$ by vehicle $k$ with a product 1

$c_{i j k l}$ is the unit shipping cost from origin $i$ to destination $j$ by vehicle $k$ with product $l$.

$x_{i j k l}$ is the shipped quantity from source $i$ to destination $j$ by vehicle $k$ with product $l$.

Figure 4 illustrates the representation of four-dimensional transportation problem with $m$ different sources and $n$ different destinations, v different vehicles and $p$ different commodities.

\subsection{Multiobjective Four-Dimensional Transportation Problem}

The mathematical representation of the multiobjective four-dimensional transportation problem that has four indices, can be represented as follows: 

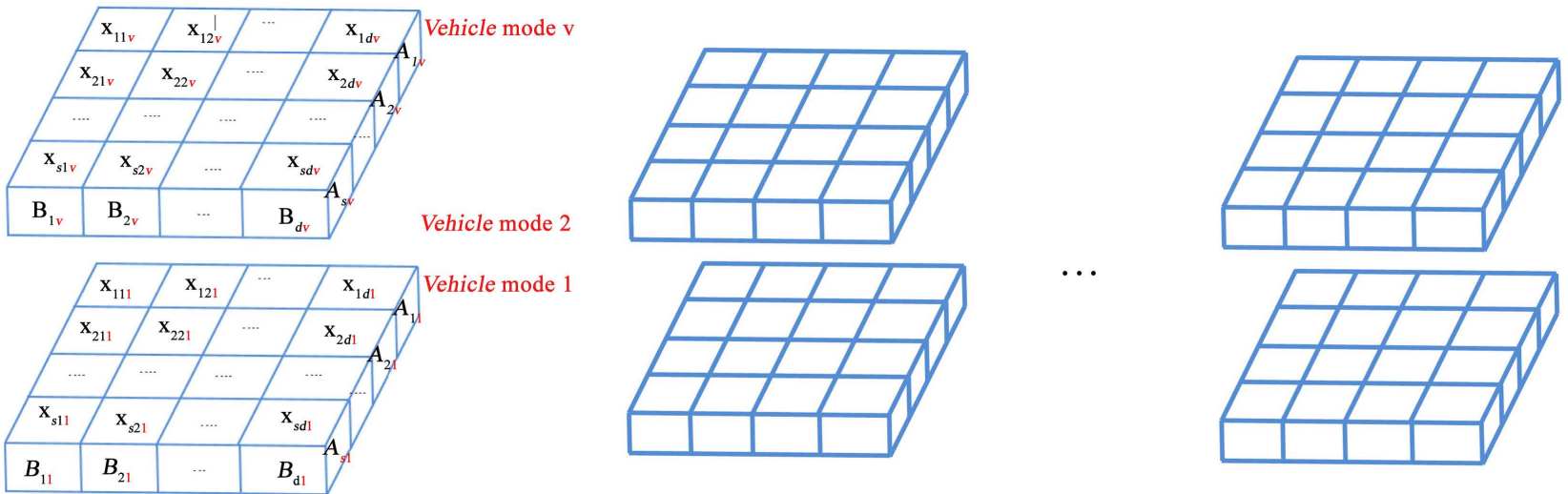

$1=1$

$1=2$

$1=p$

Figure 4. Representation of four-dimensional transportation problem with $m$ different sources and $n$ different destinations, $V$ different vehicles and $p$ different commodities.

$\operatorname{Min} \quad z=\sum_{i=1}^{s} \sum_{j=1}^{d} \sum_{k=1}^{v} \sum_{l=1}^{p} c_{i j k l} X_{i j k l}$,

subject to

$$
\begin{aligned}
& \sum_{j=1}^{d} x_{i j k l}=A_{i k l}, \quad i=1,2, \cdots, s ; k=1,2, \cdots, v ; l=1,2, \cdots, p, \\
& \sum_{i=1}^{s} x_{i j k l}=B_{j k l}, \quad j=1,2, \cdots, d ; k=1,2, \cdots, v ; l=1,2, \cdots, p, \\
& X_{i j k l} \geq 0, \quad \forall i, j, k, l \\
& \sum_{i=1}^{s} A_{i k l}=\sum_{j=1}^{d} B_{j k l}, \quad k=1,2, \cdots, v ; l=1,2, \cdots, p,
\end{aligned}
$$

\section{Relaxed Multi-Dimensional Multi-Objective Problem}

The author considers some modification of the usual multi-index multi-objective transportation problem [4], by not allowing upper bounds for the admissible capacity for the total quantity to be sent from $i^{\text {th }}$ origin to the $f^{\text {th }}$ destination.

$$
\operatorname{minimize}\left\{F_{1}=\sum_{i=1}^{m} \sum_{j=1}^{n} \sum_{k=1}^{p} C_{i j k}^{1} x_{i j k}, F_{2}=\sum_{i=1}^{m} \sum_{j=1}^{n} \sum_{k=1}^{p} C_{i j k}^{2} x_{i j k}, \cdots, F_{l}=\sum_{i=1}^{m} \sum_{j=1}^{n} \sum_{k=1}^{p} C_{i j k}^{l} x_{i j k}\right\}
$$

Subject to

$$
\begin{aligned}
& \sum_{i=1}^{m} x_{i j k}=A_{j k}, \\
& \sum_{j=1}^{n} x_{i j k}=B_{k i} \\
& x_{i j k} \geq 0 ; i=1,2, \cdots, m ; j=1,2, \cdots, n ; k=1,2, \cdots, p \\
& \sum_{j=1}^{n} A_{j k}=\sum_{i=1}^{m} B_{k i}, \quad \sum_{j=1}^{n} \sum_{k=1}^{p} A_{j k}=\sum_{k=1}^{p} \sum_{i=1}^{m} B_{k i}
\end{aligned}
$$

\section{Modified BBO Algorithm}

Biogeography Based Algorithm [16] is population-based evolutionary algorithm. 
It is based on investigation the biogeography science. The simulation of biogeography science declares the Migration of species (animals, birds, swarm), the development of species between habitat and Extinction of some species. In biogeography-based algorithm, habitat convenience Index is analogs to species island which is denoted as a candidate individual. This Habitat is known with high HSI are devoted to life. HSI corresponds to the BBO solution's goodness. Rainfall, topographic diversity, temperature, land area, vegetation diversity, and others are some aspects which are included with HSI. Suitability index variables (SIVs) are known as aspects which identify habitability. Figure 5 demonstrates a species model in a single type of island.

Habitability is a term in which SIVs is the island's independent variable and the dependent variable is HSI. High-HSI habitats are the habitat with a large number of species and Low habitats are habitats with few species. Facets of High HSI solution are given to low HSI, these facets are acquired by Low HSI provided by High HSI solution. Facets of High HSI solution is to emigrate to Low HSI solution. Emigration and Immigration tends to reform the solutions and thus emerging a solution to optimization problem. Like other evolutionary population dependent algorithms, BBO solution search procedure is an iterative procedure. After, BBO population's initialization migration and mutation are the two type of procedures which necessitates the recited iterations. The schemes are described as.

\section{1) Initialization of the population}

The arbitrarily depressed population of habitat is created by IBBO, where every population habitat $H_{i},(i=1,2, \cdots$, Population $)$ is a d-dimensional vector (the problem's variables). $H_{i}$ represents the $i^{\text {th }}$ habitat in the population. Every habitat is created using the following equations.

$$
H_{i j}=H_{\min _{i j}}+\rho\left(H_{\max _{i j}}-H_{\min _{i j}}\right)
$$

$H_{\min _{i j}}$ is the lower bound of $H_{i}$,

$H_{\text {max }_{i j}}$ is the upper bound of $H_{i}$,

$\alpha$ is the rand number, $\alpha \in[0,1]$.

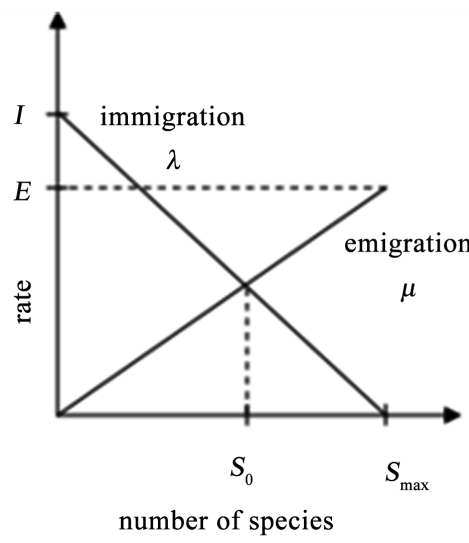

Figure 5. Species model [2]. 
In order to create the suitable design of habitat using IBBO, first, consider each solution consists of a sequence of $p$ layers (each layer has a size of $m \times n$, where $m$ is the number of origins and $n$ is the number of different destinations). Each habitat (Figure 6) consists of $p$ layers ( $p$ is the number of commodities). we generate each layer randomly such that

$$
\sum_{i=1}^{m} x_{i j k}=A_{j k}, \quad \sum_{j=1}^{n} x_{i j k}=B_{k i} \text { for layer } k
$$

E.g., in Figure 7, there are two origins $(m=2)$ and we have three destination $(n=3)$, with 2 commodities $(p=2)$. In order to design the suitable structure of the solution using IBBO, first consider each individual consists of two layers (i.e., number of commodities $p=2$ ).

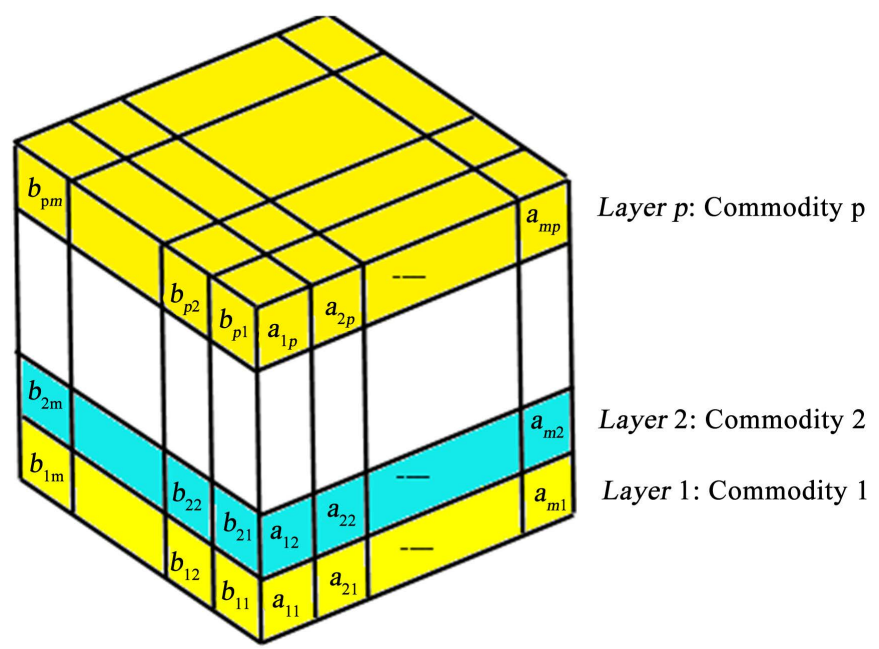

Figure 6. Structure of individual for M_MOTP with $m$ sources and $n$ destinations and $p$ commodities.

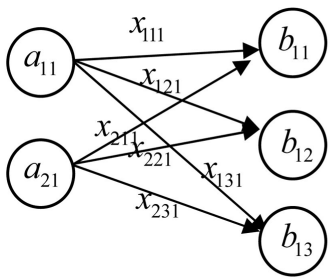

(a)

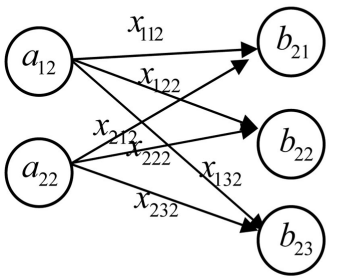

(c)

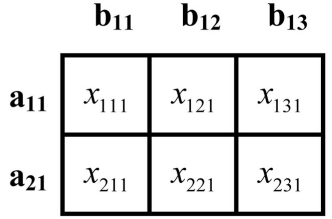

(b)

\begin{tabular}{c|l|l|l|}
\multicolumn{1}{c}{} & \multicolumn{1}{c}{$\mathbf{b}_{21}$} & \multicolumn{1}{c}{$\mathbf{b}_{22}$} & \multicolumn{1}{c}{$\mathbf{b}_{23}$} \\
\cline { 2 - 4 } $\mathbf{a}_{12}$ & $x_{112}$ & $x_{122}$ & $x_{132}$ \\
\cline { 2 - 4 } $\mathbf{a}_{22}$ & $x_{212}$ & $x_{222}$ & $x_{232}$ \\
\cline { 2 - 4 } & &
\end{tabular}

(d)

Figure 7. (a) and (b) Illustration of layer 1's representation, (a) Transportation graph, (b) solution structure; (c) and (d) Illustration of layer 2's representation; (c) Transportation graph; (d) Solution structure. 
We generate the $1^{\text {st }}$ layer (i.e., $1^{\text {st }}$ commodity) randomly such that, $x_{111}+x_{121}+x_{131}=a_{11}, \quad x_{211}+x_{221}+x_{231}=a_{21}, \quad x_{111}+x_{211}=b_{11}, \quad x_{121}+x_{221}=b_{12}$, $x_{131}+x_{231}=b_{13}$.

For the $2^{\text {nd }}$ layer (i.e., $2^{\text {nd }}$ commodity) randomly such that, $x_{112}+x_{122}+x_{132}=a_{12}, \quad x_{212}+x_{222}+x_{232}=a_{22}, \quad x_{112}+x_{212}=b_{21}, \quad x_{122}+x_{222}=b_{22}$, $x_{132}+x_{232}=b_{23}$.

From studying the transportation problem, it is obvious to note that all generated solution has the following distinguished features.

a) All of generated habitats are feasible.

b) The habitat length is only $m \times n \times p$, that is $\sum_{i=1}^{m} x_{i j k}=a_{j k} \forall j=1,2, \cdots, n$, $\sum_{j=1}^{n} x_{i j k}=b_{i k} \forall i=1,2, \cdots, m$, for each $k$ layer, where the problem has $m$ sources and $n$ destinations and $p$ commodities.

\section{2) Migration}

By taking advantage of the emigration rate $\left(\mu_{j}\right)$ as well as immigration rate $\left(\lambda_{i}\right)$ facets are probabilistically shared between the habitats this procedure is known as Migration [17]. To bestowing the facets between candidate solution for modifying goodness the migration operator is liable. According to the probability of $\left(\mu_{j}\right)$ and $\left(\lambda_{i}\right)$ emigration solution and immigration solutions are selected respectively.

\section{3) Mutation}

In BBO algorithm solution's variety are keeping the mutation [18] is culpable. For low and high HSI candidate solutions mutation renders a possibility for improving the solution's goodness. It is able to intensify the solution's quality even if they have more innumerable solutions already.

\section{4) Pseudo-Code of the IBBO Algorithm}

From the above discussion in Section 2, BBO's pseudo-code is depicted in Figure 8.

\section{5) Dominance Criteria}

To improve the IBBO to deal with multiple objective optimization problems, the dominance criteria implemented [19] [20] was in the proposed algorithm, which presented as follows:

For any optimization problem that has more than one objective function $k>1$ (i.e., $\left.f_{j}, j=1, \cdots, k\right)$ any two candidate solution $x^{1}$ and $x^{2}$ could have one of two situations, "one dominates the other" or "none dominates the other".

A solution $x^{1}$ dominates the solution $x^{2}$, if the following two conditions are verified [21].

"the operator $\prec$ refers to worse and the operator $\succ$ refers to better".

a) The solution $x^{1}$ is no worse than the solution $x^{2}$ in all objectives.

b) The solution $x^{1}$ is strictly better than the solution $x^{2}$ in at least one objective function (i.e., $f_{j}\left(x^{1}\right) \succ f_{j}\left(x^{2}\right)$ for at least one $j \in\{1,2, \cdots, k\}$ ).

If any of these two conditions is violated, the solution $x^{1}$ does not dominate the solution $x^{2}$. This algorithm is repeatedly for all solutions, all solutions that 


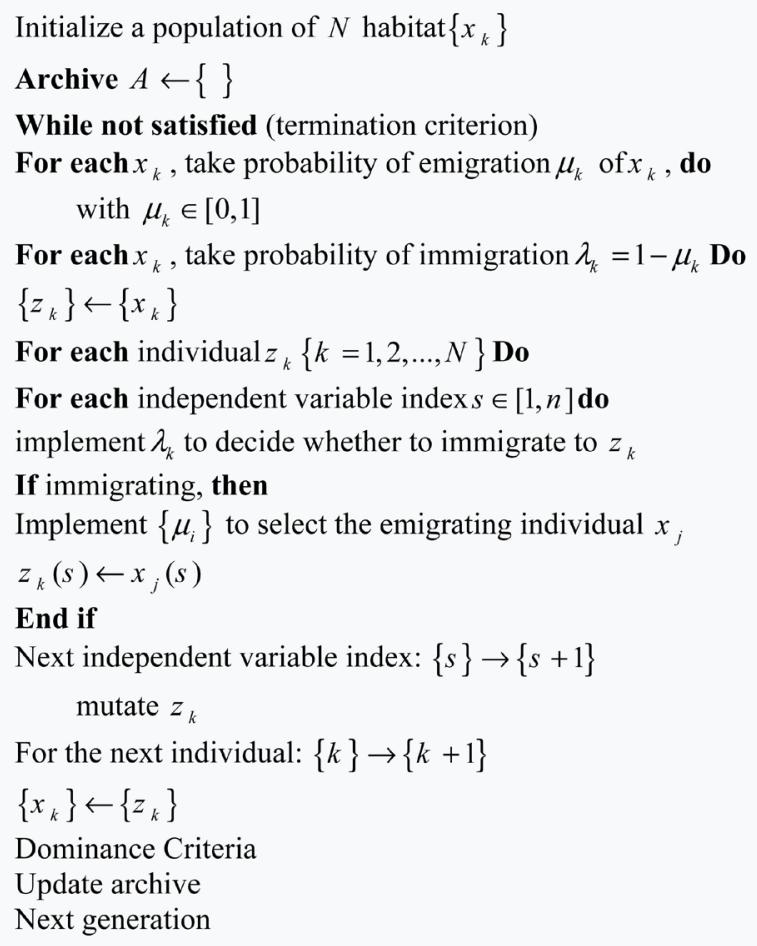

Figure 8. Pseudo code of the proposed algorithm.

are not dominated by any other solutions are constitute "the nondominated set".

\section{6) Archive Algorithm}

The goal of the archive function [21] is to collect a new set of solutions in each iteration counter $t$, it uses the solutions in the old archive to update the solutions in the archive pool, see Figure 9. In general, the goal of this archive is to collect useful data about the underlying optimization problem during the run and update the content of the stored data (current archive content) see Figure 1.

\section{7) Elitist Strategy}

In order to guarantee rapid convergence to the nondominated-optimal solutions, elitist strategy could be implemented in the algorithm procedure. So, we propose an archiving algorithm that ensures the progress towards the nondominated-optimal solutions and at the same time covering of the whole range of the found nondominated solutions.

\section{Experimental, Results and Discussions}

The proposed algorithm was implemented using MATLAB version 7.0 (R14/2004). To ensure the effectiveness of the method on the multidimensional transportation problem, a numerical example was used in the computational studies.

\section{Real application in the North Western Coastal Strip of Egypt}

The following topological shape of the North Western Coastal Strip of Egypt (Figure 1) presents the geographical phenomena in a simplified manner, and does not mean the representation of all its characteristics and geographical relations; it abstracts them from some aspects that help to simplify them [3]. The 


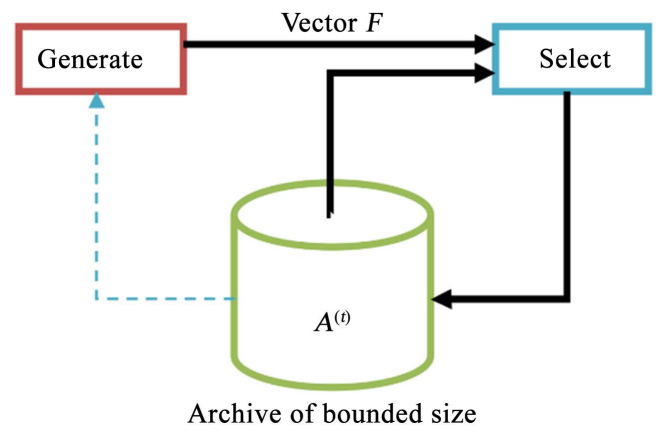

Figure 9. Block structure of archive-based selection algorithm.

transformation of the north-west coast road network into a topological form is intended to facilitate its analysis and to make conclusions that can predict what its future status will be. The topological shape [10] consists of the vertices or nodes. The main nodes, the Edges or Arcs, are the roads or direct links between the nodes. The contract has gained importance for its privileged position on the network.

Table 1 gives the main roads which were investigated in our study. Figure 10 gives the daily cargo flows on the Alexandria International Highway Salloum and some major and regional roads in the Northwest Coast 2014 [3]. The movement of goods transport and circulation is related to the study of commercial activity and its impact on transport, which mean that the role of transport and its impact on trade in the study area and the quality of goods and cargo are classified to:

Outbound trade: Carriages are transported by quarry ore, limestone, sand, gravel and child from quarries located in the province to the rest of the Republic. On the other hand, the trucks transport the production of dates, olives and fish in the province to the rest of the Republic.

Trade Imports: Products, petroleum products and water represent the most important commodities imported to the region from factories and factories. Imports also include cement. In addition, a large proportion of imports.

The nature of movable materials requires the use of a certain method, not the other, as in the case of liquid or flammable materials. Table 2 shows the movement rate of transport of goods, when examining the quality of transport between the destinations of the movement. Transport of goods on roads along the northwestern coastline.

The effectiveness of the proposed algorithm will be illustrated on the numerical example, taken from [4] for which $m=4, n=3, P=2$.

The $1^{\text {st }}$ objective cost matrix $C^{1}(i, j, k)$ is as follows:

\begin{tabular}{llllllll}
\hline \multirow{3}{*}{$C^{1}(i, j, 1)$} & 4 & 3 & 5 & 8 & 6 & 3 \\
& 8 & 6 & 2 & $C^{1}(i, j, 2)$ & 5 & 4 & 1 \\
& 7 & 4 & 1 & 9 & 2 & 6 \\
\hline
\end{tabular}




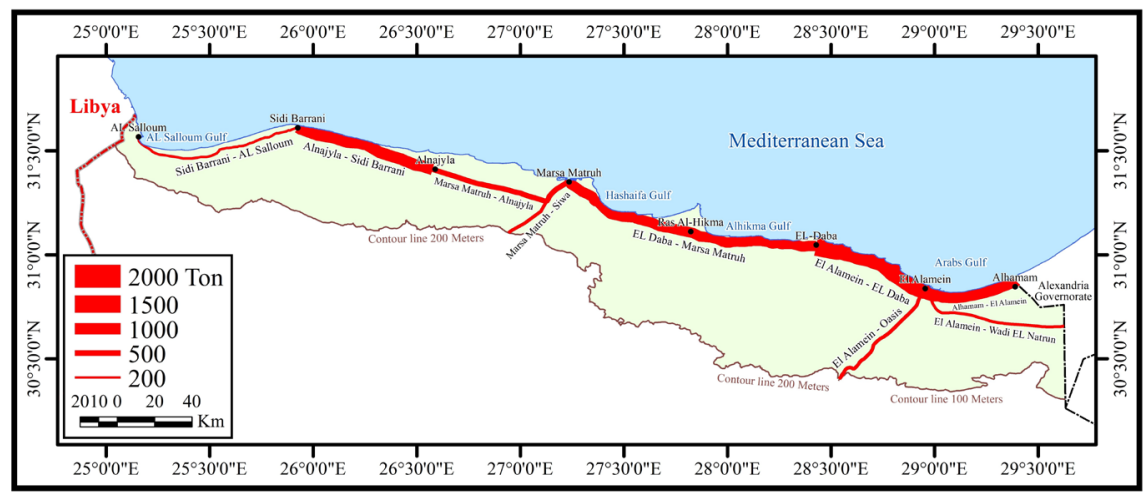

Figure 10. Daily cargo flows on the Alexandria International Highway Salloum and some major and regional roads in the Northwest Coast 2014 [3].

Table 1. The main roads in the northwestern coastal strip of Egypt.

\begin{tabular}{c}
\hline Main roads \\
\hline Iinternational coastal road \\
Al-Alamein Road and Wadi Al-Natroun \\
Al-Alamain Road \\
Siwa Matruh Road \\
\hline
\end{tabular}

Table 2. The type of goods transported on the roads.

\begin{tabular}{c} 
Type of goods transferred \\
Food and nutrition \\
Agricultural \& Animal Products \\
Marble, quarry products and crushers \\
Petroleum products \\
Waters \\
\hline
\end{tabular}

The $2^{\text {nd }}$ objective cost matrix $C^{1}(i, j, k)$ is as follows:

\begin{tabular}{|c|c|c|c|c|c|c|c|}
\hline \multirow{4}{*}{$C^{2}(i, j, 1)$} & 5 & 6 & 7 & \multirow{4}{*}{$C^{2}(i, j, 2)$} & 10 & 9 & 9 \\
\hline & 4 & 5 & 2 & & 7 & 9 & 2 \\
\hline & 7 & 4 & 1 & & 8 & 7 & 9 \\
\hline & 9 & 10 & 12 & & 8 & 4 & 5 \\
\hline
\end{tabular}

The capacity of the $I^{\text {th }}$ plant (source) is $S(i, p)$ and the requirement of the $f^{\text {th }}$ warehouse (destination) is $D(j, p)$ for different commodity is as follows:

\begin{tabular}{ccccccc}
\hline & 9 & 6 & & & & \\
$S(i, p)$ & 14 & 7 & $D(i, p)$ & & & \\
& 6 & 5 & 5 & 8 & 11 \\
& 7 & 6 & & & \\
\hline
\end{tabular}




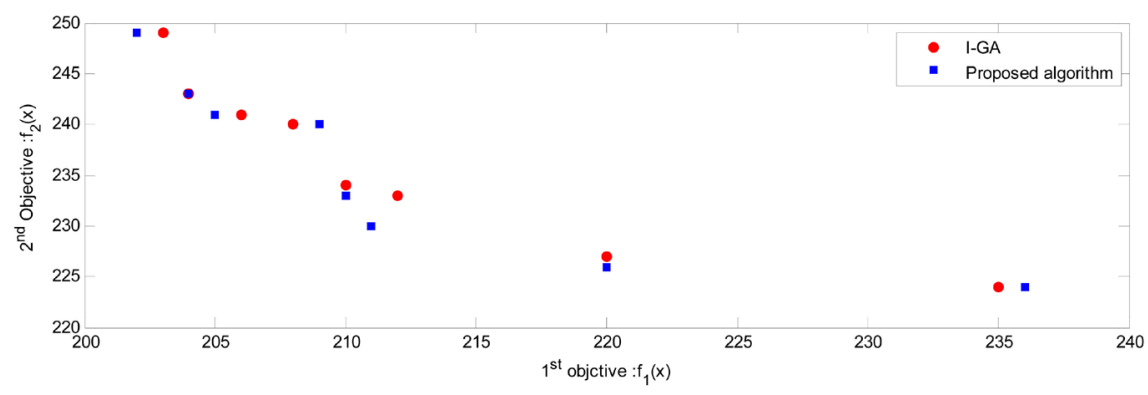

Figure 11. The detected Pareto optimal solution.

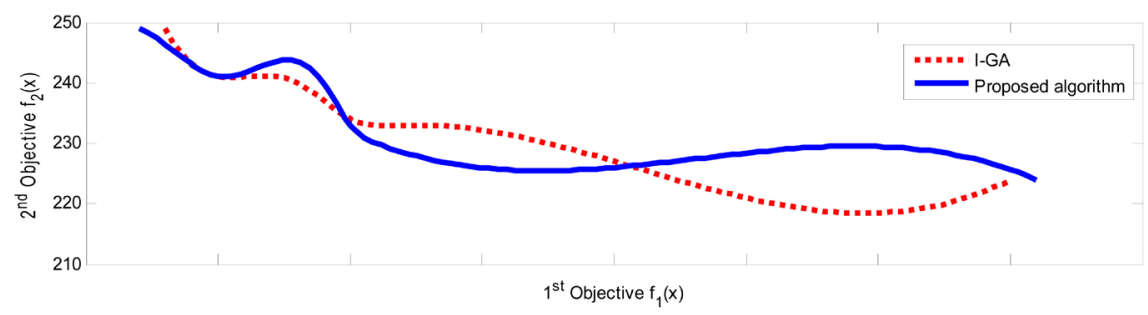

Figure 12. Curve fitted Pareto optimal solution.

Figure 11 shows the results obtained by proposed approaches versus the results obtained from I-GA [4], the results declare the validity of the proposed algorithm, the set of points that dominate the obtained solutions obtained by I-GA [4]. IBBO dominate $60 \%$ of points of I-GA, on the other hand I-GA dominate $40 \%$ of points of IBBO, which illustrates the superiority of the proposed algorithm. Figure 12 represents curve fitted Pareto optimal solution, which helps the decision maker to make the right decision and select the best compromise alternative.

\section{Conclusions}

In this research, we present an improved evolutionary algorithm called improved biogeography-based optimization algorithm (IBBO) for solving multiobjective multi-dimensional transportation problem with an application to flow of the commodities of the main roads to main nodes in the North Western Coastal Strip of Egypt. Finally, we report numerical simulation on real application in order to assess its performances and convergence, also to set up the actual simulation burden of the proposed algorithm. The proposed method implements the concept of dominance criteria to deal with the multiple objective functions, which enable the decision maker to detect the set of all Pareto frontier of the problem which has a large-scale size. The main characteristics features of the proposed IBBO could be described as follows:

1) The detected Pareto optimal solutions are well distributed and have well satisfactory diversity characteristics.

2) Numerical results verified the superiority and the capability of the proposed algorithm.

3) This approach could also be used to treat many real applications in trans- 
portation network and urban planning.

In the future work the risk analysis will be investigated, including the best and safe road to transportation.

\section{Conflicts of Interest}

The authors declare no conflicts of interest regarding the publication of this paper.

\section{References}

[1] Mousa, A.A. (2010) Using Genetic Algorithm and TOPSIS Technique for Multiobjective Transportation Problem: A Hybrid Approach. International Journal of Computer Mathematics, 87, 3017-3029. https://doi.org/10.1080/00207160902875262

[2] Rodrigue, J.-P. and Ducruet, C. (2017) The Geography of Transportation Networks. Routledge, New York, 440 p.

[3] Abokila, T. (2017) The Role of Roads in Developing the North Western Coastal Strip of Egypt: An Analytical Study Employing GIS. PhD Thesis, Banha University, Banha.

[4] El-Shorbagy, M.A., Mousa, A.A.A., ALoraby, H. and Abo-Kila, T. (2020) Evolutionary Algorithm for Multi-Objective Multi-Index Transportation Problem under Fuzziness. Journal of Applied Research on Industrial Engineering, 7, 36-56.

[5] Skitsko, V. and Voinikov, M. (2020) Solving Four-Index Transportation Problem with the Use of a Genetic Algorithm. LogForum, 16, Article No. 6. https://doi.org/10.17270/J.LOG.2020.493

[6] An, O., Chen, X., Li, Y., Han, Y., Zhang, J. and Shi, H. (2021) An Improved Non-Dominated Sorting Biogeography-Based Optimization Algorithm for the (Hybrid) Multi-Objective Flexible Job-Shop Scheduling Problem. Applied Soft Computing, 99, Article ID: 106869. https://doi.org/10.1016/j.asoc.2020.106869

[7] Tu, J., Chen, H., Liu, J., Asghar Heidari, A., Zhang, X., Wang, M., Ruby, R. and Pham Q.-V. (2021) Evolutionary Biogeography-Based Whale Optimization Methods with Communication Structure: Towards Measuring the Balance. Knowledge-Based Systems, 212, Article ID: 106642. https://doi.org/10.1016/j.knosys.2020.106642

[8] Mirjalili, S. (2019) Biogeography-Based Optimisation. In: Mirjalili, S., Ed., Evolutionary Algorithms and Neural Networks, Vol. 780, Springer, Cham, 57-72. https://doi.org/10.1007/978-3-319-93025-1 5

[9] Ma, H. and Simon, D. (2011) Blended Biogeography-Based Optimization for Constrained Optimization. Engineering Applications of Artificial Intelligence, 24, 517-525. https://doi.org/10.1016/j.engappai.2010.08.005

[10] Arlinghaus, S.L., Arlinghaus, W.C. and Harary, F. (2001) Graph Theory and Geography: An Interactive View. John Wiley, New York.

[11] Hamdy, T. (2016) Operations Research: An Introduction. 10th Edition, Pearson Publisher, London.

[12] Osman M.S., Abo-Sinna, M.A. and Mousa, A.A. (2006) IT-CEMOP: An Iterative Co-Evolutionary Algorithm for Multiobjective Optimization Problem with Nonlinear Constraints. Journal of Applied Mathematics \& Computation, 183, 373-389. https://doi.org/10.1016/j.amc.2006.05.095 
[13] Kasana, H.S. and Kumar, K.D. (2003) Introductory Operations Research, Theory and Applications. Springer-Verlag, Berlin.

[14] Haley, K.B. (1963) The Multi-Index Problem. Operations Research, 11, 368-379. https://doi.org/10.1287/opre.11.3.368

[15] Abd Elazeem, A.E.M., Mousa, A.A.A., El-Shorbagy, M.A., Elagan, S.K. and Abo-Elnaga, Y. (2021) Detecting All Non-Dominated Points for Multi-Objective Multi-Index Transportation Problems. Sustainability, 13, Article No. 1372. https://doi.org/10.3390/su13031372

[16] Simon, D. (2008) Biogeography-Based Optimization. IEEE Transactions on Evolutionary Computation, 12, 702-713. https://doi.org/10.1109/TEVC.2008.919004

[17] Ma, H. (2010) An Analysis of the Equilibrium of Migration Models for Biogeography-Based Optimization. Information Sciences, 180, 3444-3464. https://doi.org/10.1016/j.ins.2010.05.035

[18] Farrokh Ghatte, H. (2021) A Hybrid of Firefly and Biogeography-Based Optimization Algorithms for Optimal Design of Steel Frames. Arabian Journal for Science and Engineering, 46, 4703-4717. https://doi.org/10.1007/s13369-020-05118-w

[19] Farag, M.A., El-Shorbagy, M.A. Mousa, A.A. and El-Desoky, I.M. (2020) A New Hybrid Metaheuristic Algorithm for Multiobjective Optimization Problems. International Journal of Computational Intelligence Systems, 13, 920-940. https://doi.org/10.2991/ijcis.d.200618.001

[20] Osman, M.S., Abo-Sinna, M.A. and Mousa, A.A. (2009) A Epsilon-Dominance Based Multiobjective Genetic Algorithm for Economic Emission Load Dispatch Optimization Problem. Electric Power Systems Research, 79, 1561-1567. https://doi.org/10.1016/j.epsr.2009.06.003

[21] Mousa, A.A. and Elattar, E.E. (2014) Best Compromise Alternative to EELD Problem Using Hybrid Multiobjective Quantum Genetic Algorithm. Applied Mathematics \& Information Sciences, 8, 2889-2902. https://doi.org/10.12785/amis/080626 